\title{
Shape transitions in proton-neutron systems
}

\author{
Serdar Kuyucak ${ }^{1}$ \\ Department of Theoretical Physics, Research School of Physical Sciences, \\ Australian National University, Canberra, ACT 0200, Australia \\ Ka-Hae Kim² and Takaharu Otsuka ${ }^{3}$
Department of Physics, University of Tokyo, Hongo, Tokyo 113, Japan
}

\begin{abstract}
We explore the possibility of shape transitions in proton-neutron systems driven by deformation differences between proton and neutron fluids. Within the framework of the proton-neutron interacting boson model, we show that such dynamic shape transitions cannot occur in well deformed nuclei but are a possibility in transitional nuclei. Likely candidates in the Os-Pt isotopes are discussed and predictions of the model are compared with the existing data.
\end{abstract}

PACS: 21.60.Ev, 21.60.Fw, 23.20.Js, 27.80.+w

Keywords: Interacting boson model; Proton-neutron deformations; Shape transition; Quadrupole moments; Transitional Nuclei

${ }^{1}$ E-mail: sek105@phys.anu.edu.au

${ }^{2}$ E-mail: kae@nt.phys.s.u-tokyo.ac.jp

${ }^{3}$ E-mail: otsuka@phys.s.u-tokyo.ac.jp 
Studies of shape transitions in nuclei at low excitation energies have been mostly based on coexistence of different shell model configurations [1]. In collective models, shape transitions could also arise from the competition between two macroscopic shape variables. An example of such a shape transition, arising from the competition between the quadrupole and hexadecapole shapes, was given in the $s d g$ boson model earlier [2]. Two-fluid models, where proton and neutron degrees of freedom are treated separately, could furnish another example. Measurements of neutron deformation using pion beams [3] indicate that there could be sizable differences between the proton and neutron deformations. There is also indirect evidence from g-factor measurements [4] that the quadrupole operators for protons and neutrons could be rather different, especially in transitional nuclei [5]. In this letter, we investigate in the framework of the proton-neutron interacting boson model (IBM-2) [6, 4] whether such differences could also drive a shape transition in proton-neutron systems. For this purpose we use the analytic $1 / N$ expansion method [8] and the exact numerical diagonalization results from the code NPBOS [9]. The $1 / N$ expansion is based on angular momentum projected mean field theory and therefore provides an intuitive picture for shape transitions. Further, due to their analytic formulation, a global study of the parameter space can be easily carried out, which shows that transitional nuclei provide the most likely candidates for such a dynamic shape transition. As the $1 / N$ expansion results are not very reliable for transitional nuclei, we use exact diagonalization in exhibiting the shape transition and applications in this region.

We employ the simplest IBM-2 Hamiltonian suggested by microscopics [6, 7]

$$
H=\epsilon_{\pi} \hat{n}_{d \pi}+\epsilon_{\nu} \hat{n}_{d \nu}-\kappa Q_{\pi} \cdot Q_{\nu}+\xi M
$$

where $\hat{n}_{d \rho}, \rho=\pi, \nu$ are the $d$-boson number operators for proton and neutron bosons, $M$ is the Majorana operator in Casimir form and $Q_{\rho}$ are the quadrupole operators given by

$$
Q_{\rho}=\left[d_{\rho}^{\dagger} s_{\rho}+s_{\rho}^{\dagger} \tilde{d}_{\rho}\right]+\chi_{\rho}\left[d_{\rho}^{\dagger} \tilde{d}_{\rho}\right]^{(2)}, \quad \rho=\pi, \nu
$$

The E2 matrix elements (m.e.) are calculated using the operator

$$
T(E 2)=e_{\pi} Q_{\pi}+e_{\nu} Q_{\nu}
$$


where $e_{\pi}, e_{\nu}$ are effective charges, and the same quadrupole operators (2) are used as in the Hamiltonian. For future reference, we introduce $F$-spin scalar and vector parameters as

$$
\begin{gathered}
\epsilon_{\mathrm{s}}=\left(\epsilon_{\pi}+\epsilon_{\nu}\right) / 2, \quad \epsilon_{\mathrm{v}}=\epsilon_{\pi}-\epsilon_{\nu} \\
\chi_{\mathrm{s}}=\left(\chi_{\pi}+\chi_{\nu}\right) / 2, \quad \chi_{\mathrm{v}}=\chi_{\pi}-\chi_{\nu} .
\end{gathered}
$$

Although other terms are sometimes included in detailed IBM-2 studies, the Hamiltonian (11) is found to give an adequate description of level energies and electromagnetic (E2 and M1) transitions [7].

The $1 / N$ expansion solutions follow from using the boson condensate

$$
\left|N_{\pi}, N_{\nu}\right\rangle=\left(N_{\pi} ! N_{\nu} !\right)^{-1 / 2}\left(b_{\pi}^{\dagger}\right)^{N_{\pi}}\left(b_{\nu}^{\dagger}\right)^{N_{\nu}}|0\rangle, \quad b_{\rho}^{\dagger}=\left(1+\beta_{\rho}^{2}\right)^{-1 / 2}\left(s_{\rho}^{\dagger}+\beta_{\rho} d_{\rho 0}^{\dagger}\right),
$$

as a trial state in a variation after projection (VAP) calculation. Here $\beta_{\pi}$ and $\beta_{\nu}$ are the mean field deformations for the proton and neutron fluids, which are determined from the expectation value of the Hamiltonian (1)

$$
E_{L}=\left\langle N_{\pi}, N_{\nu}\left|H P_{00}^{L}\right| N_{\pi}, N_{\nu}\right\rangle /\left\langle N_{\pi}, N_{\nu}\left|P_{00}^{L}\right| N_{\pi}, N_{\nu}\right\rangle
$$

by the VAP procedure. In Eq. (6), $P_{00}^{L}$ denotes the angular momentum projection operator. Note that the IBM deformation variables involve only the valence nucleons, and therefore they are much larger $(\beta \sim 1-1.4)$ than the typical geometrical model values $(\beta \sim 0.2-0.3)[10$. We refer to Ref. [8] for details of the $1 / N$ expansion method, and quote here the energy expression obtained from Eq. (6) for the Hamiltonian (1). To the leading order in $1 / N$, which is sufficient for discussion of systematic features, the energy surface and moment of inertia terms are given by

$$
\begin{aligned}
E_{L}= & E_{0}+C_{L} L(L+1), \\
E_{0}= & \sum_{\rho} \frac{\varepsilon_{\rho} N_{\rho} \beta_{\rho}^{2}}{1+\beta_{\rho}^{2}}-\kappa N_{\pi} N_{\nu} \beta_{\pi} \beta_{\nu} \frac{\left(2+\bar{\chi}_{\pi} \beta_{\pi}\right)\left(2+\bar{\chi}_{\nu} \beta_{\nu}\right)}{\left(1+\beta_{\pi}^{2}\right)\left(1+\beta_{\nu}^{2}\right)}+\frac{\xi N_{\pi} N_{\nu}\left(\beta_{\pi}-\beta_{\nu}\right)^{2}}{\left(1+\beta_{\pi}^{2}\right)\left(1+\beta_{\nu}^{2}\right)}, \\
C_{L}= & \frac{1}{(a N)^{2}}\left\{(6-a) \sum_{\rho} \frac{\varepsilon_{\rho} N_{\rho} \beta_{\rho}^{2}}{1+\beta_{\rho}^{2}}\right. \\
& +\kappa \frac{N_{\pi} N_{\nu} \beta_{\pi} \beta_{\nu}}{\left(1+\beta_{\pi}^{2}\right)\left(1+\beta_{\nu}^{2}\right)}\left(8 a-12+(4 a-12)\left(\bar{\chi}_{\pi} \beta_{\pi}+\bar{\chi}_{\nu} \beta_{\nu}\right)+(2 a-9) \bar{\chi}_{\pi} \bar{\chi}_{\nu} \beta_{\pi} \beta_{\nu}\right) \\
& \left.+2 \xi \frac{N_{\pi} N_{\nu}\left(1+\beta_{\pi} \beta_{\nu}\right)}{\left(1+\beta_{\pi}^{2}\right)\left(1+\beta_{\nu}^{2}\right)}\left(a+(a-6) \beta_{\pi} \beta_{\nu}\right)\right\},
\end{aligned}
$$


where $\bar{\chi}_{\rho}=-\sqrt{2 / 7} \chi_{\rho}$. The quantity " $a$ " represents the average "angular momentum squared" carried by a single boson and is given by

$$
a=\frac{6}{N}\left(\frac{N_{\pi} \beta_{\pi}^{2}}{1+\beta_{\pi}^{2}}+\frac{N_{\nu} \beta_{\nu}^{2}}{1+\beta_{\nu}^{2}}\right) .
$$

In Fig. 1, we show the contour plots of the energy surface $E_{0}$ obtained from Eq. (8) in a typical deformed (top) and transitional (bottom) nucleus. The positive $\beta$ values correspond to prolate and the negative ones to oblate deformations. The prolate (absolute) minima are in the first quadrant and the oblate ones are in the third. There are 20 contour lines at $1 \mathrm{MeV}$ steps, thus the maxima in the second quadrant are about $20 \mathrm{MeV}$ high. In the deformed case, the prolate minimum in the first quadrant $\left(E_{\min }=-4.83 \mathrm{MeV}\right)$ is well separated from the oblate one in the third quadrant with an energy difference of $\Delta E=2.23 \mathrm{MeV}$. Including the moment of inertia term (9) in the energy surface, this energy difference gets larger with increasing spin. Thus there is no chance of a cross over from the prolate to the oblate minimum. Although we have used a particular parametrization here for illustration purposes, in fact, this is a general feature of all deformed nuclei as can be verified by systematic studies of Eqs. (7-9). Since such a study is already available for IBM-1 [2], and the IBM-2 results are very similar to those of IBM-1 for deformed nuclei, we will not elaborate on them further.

In the transitional case (bottom), the absolute minimum is at $E_{\min }=-3.07$ $\mathrm{MeV}$, and the energy difference between the two minima is much smaller $(\Delta E=0.85$ $\mathrm{MeV}$ ). Furthermore, the moment of inertia in the oblate minimum could be larger than that in the prolate one depending on the choice of the vector parameters in Eq. (4). Hence the energy difference between the two minima could decrease with increasing spin, with possibility of a cross over at some critical spin. Unfortunately, due to the soft energy surface, the $1 / N$ expansion formulas are not very reliable for transitional nuclei, and a quantitative study of shape transitions is not possible using the analytic formulas (7-9) in this region.

In order to exhibit the proposed proton-neutron shape transition and to study general conditions for its existence, we therefore rely on numerical diagonalization. The signature for shape change is taken from the yrast spectroscopic quadrupole moments, which are negative for a prolate shape and positive for an oblate one. Since 
the shape change is driven by differences in the proton and neutron deformations, the critical quantities to study are the $F$-spin vector parameters, $N_{\mathrm{v}}=N_{\pi}-N_{\nu}, \chi_{\mathrm{v}}$ and $\epsilon_{\mathrm{v}}$, which can induce asymmetries between the proton and neutron variables. In principle, the Majorana interaction could also have an influence on the protonneutron asymmetry. However, variations in the Majorana strength (of order 20-30\%) did not lead to any discernible effect on shape change. In Fig. 2, we show the effect of these three vector parameters on yrast quadrupole moments in a typical prolate transitional nucleus with $F$-spin scalar parameters, $N=12, \epsilon_{\mathrm{s}}=0.4 \mathrm{MeV}, \kappa=0.15$ $\mathrm{MeV}, \xi=0.17 \mathrm{MeV}$, and $\chi_{\mathrm{s}}=-0.2$. The boson effective charges are taken as $e_{\pi}=e_{\nu}=0.1 \mathrm{eb}$. Fig. 2a shows the $N_{\mathrm{v}}$ dependence of the quadrupole moments $Q(L)$ for $\chi_{\mathrm{v}}=2$ and $\epsilon_{\mathrm{v}}=0$. It is seen that $Q(L)$ changes sign between the spins $L=16-18$ in the case of $N_{\mathrm{v}}=4$, but remains prolate in the other two cases. Fig. 2b shows a similar study of $\chi_{\mathrm{v}}$ dependence of $Q(L)$ for $N_{\mathrm{v}}=4$ and $\epsilon_{\mathrm{v}}=0$. Note that the bottom results in Figs. $2 \mathrm{a}$ and $2 \mathrm{~b}$ are identical due to the symmetry of the Hamiltonian under the simultaneous interchange of $N_{\pi}-N_{\nu}$ and $\chi_{\pi}-\chi_{\nu}$. Again only in the case of $\chi_{\mathrm{v}}=2$, a shape change occurs. Finally, Fig. 2c shows the effect of $\epsilon_{\mathrm{v}}$ on the favourable case with $N_{\mathrm{v}}=4$ and $\chi_{\mathrm{v}}=2$. Compared to $N_{\mathrm{v}}$ and $\chi_{\mathrm{v}}$, $\epsilon_{\mathrm{v}}$ plays a marginal role. Nevertheless, it could shift the transition spin by several units in either direction depending on its sign; $\epsilon_{\mathrm{v}}<0$ makes the shape transition occur earlier while $\epsilon_{\mathrm{v}}>0$ retards it. The opposite is true when $N_{\mathrm{v}}$ and $\chi_{\mathrm{v}}$ are both negative.

This systematic study indicates that an essential requirement for a dynamic prolate-oblate shape transition to occur is the coherence of the two vector parameters $N_{\mathrm{v}}$ and $\chi_{\mathrm{v}}$, that is they must have the same sign. The $\epsilon_{\mathrm{v}}$ parameter with an opposite sign to the others could also facilitate the shape transition though its effect is less important. To understand these features better, we show in Fig. 3 the individual proton and neutron contributions to the yrast quadrupole moments in the favourable $\left(\chi_{\mathrm{v}}=2\right)$ and the unfavourable $\left(\chi_{\mathrm{v}}=-2\right)$ cases in Fig $2 \mathrm{~b}$. In the favourable case (top), the neutron contribution changes little from its original prolate value with increasing spin, while the proton contribution sharply increases, changing sign at spin $L=12$. At low-spins, the $(s d)$ term in the quadrupole op- 
erator (2) dominates the m.e. and it has the same (negative) sign for protons and neutrons. With increasing spin, however, the expectation value of the number of $d$-bosons in the states, and hence the m.e. of the $(d d)$ term in $(2)$ increases while that of $(s d)$ term decreases. The sign of the quadrupole moment coming from the $(d d)$ part is determined by the sign of $\chi_{\rho}$ which is positive for protons and negative for neutrons. Hence the $(s d)$ and $(d d)$ terms tend to cancel out for protons but add up for neutrons, which explains the behaviour of the individual proton and neutron quadrupole moments. The sign change in $Q(L)$ then follows from the fact that there are more protons than neutrons. In the unfavourable case (bottom), $\chi_{\pi}$ and $\chi_{\nu}$ are interchanged, thus protons favour the prolate shape and neutrons the oblate one in high-spin states (the ground state remains prolate). The behaviour of the individual contributions follow from the same reasoning. Since there are more protons, they dominate the quadrupole moment, and hence the nucleus stays in prolate shape.

The beneficial effect of $\epsilon_{\mathrm{v}}<0$ in hastening the shape transition can be understood in a similar manner. It increases the deformation of protons and reduces that of neutrons, leading to a faster change in proton contribution to $Q(L)$ and reducing the contribution of neutrons. In the more intuitive picture of Fig. 1 (bottom), including $\epsilon_{\mathrm{v}}=-0.4 \mathrm{MeV}$ in the Hamiltonian, reduces the energy difference $\Delta E$ between the two minima from $0.85 \mathrm{MeV}$ to $0.68 \mathrm{MeV}$, hence making the cross-over from the prolate minimum to the oblate one easier.

These results for prolate transitional nuclei can be readily extended to oblate ones by noting that changing the sign of both $\chi_{\pi}$ and $\chi_{\nu}$ changes the sign of the quadrupole moments but their absolute values remain the same. Thus in this case, dynamic oblate-prolate shape transitions would be possible when $N_{\mathrm{v}}$ and $\chi_{\mathrm{v}}$ have the opposite signs. The effect of $\epsilon_{\mathrm{v}}$ on $Q(L)$ remains similar; for $N_{\mathrm{v}}>0, \epsilon_{\mathrm{v}}<0$ pushes the shape transition to an earlier spin, while for $N_{\mathrm{v}}<0, \epsilon_{\mathrm{v}}>0$ does the same.

We use the above criteria in searching for a potential candidate among the transitional nuclei that might exhibit such a dynamic shape transition. The $F$-spin vector parameters in Os-Pt isotopes were recently determined from a study of $M 1$ properties, resolving the long standing anomalies observed in the $g$-factors of these 
nuclei [5]. Since $N_{\mathrm{v}}<0$ in all the isotopes considered in Ref. [5], a negative value of $\chi_{\mathrm{v}}$ in Os isotopes and a positive one in Pt isotopes is a necessary condition for a dynamic shape transition. In addition, a positive value of $\epsilon_{\mathrm{v}}$ would help it to occur at an earlier spin. Inspection of Table 1 in Ref. [5] shows that ${ }^{192}$ Os provides the most favourable case for a prolate-oblate shape transition. In Fig. 4, we show the level energies, E2 transition m.e. and quadrupole moments in the ground band of ${ }^{192}$ Os, obtained using the same parameters as in Ref. [5]. Although the calculated quadrupole moments do not actually change sign, they depict the same rapid change seen in Fig. 3, and come very close to doing so. The failure is due to the relatively low boson number $(N=8)$, which causes the boson cutoff effect to kick in early. The data on E2 transitions (Fig. 4) follow the axial rotor results closely, and do not show any sign of boson cutoff. Thus the premature decrease in the E2 m.e. is really a problem of the $s d$-IBM, and for a proper description of high-spin states one needs to include $g$-bosons in the basis. Unfortunately, an exact $s d g$-IBM-2 calculation for this nucleus is not feasible at present due to the large basis space. An approximate calculation in a truncated basis space is possible but, as a recent study indicates [12], truncation leads to unreliable results for spins $L>2 N$. This is exactly where the $s d g$-IBM-2 results are needed, therefore truncated calculations will not serve a useful purpose for extending the present results to higher spins. Nevertheless, both the proton-neutron deformation difference and the hexadecapole effects drive the system in the same direction (from prolate to oblate shape), and inclusion of $\mathrm{g}$ bosons could only enhance the shape transition. Thus combination of the present $s d$-IBM-2 results with the earlier $s d g$-IBM-1 ones [2, 13], strongly suggests a shape transition in the spin range $L=10$ to 20 for ${ }^{192}$ Os. The measured quadrupole moments deviate markedly from the axial rotor results, but to ascertain whether they actually decrease in absolute value would require more accurate measurements.

In the absence of any other guiding principle, we used the vector parameters that were determined from the $M 1$ properties in our search for shape transitions. While the $F$-spin breaking mechanism in the IBM-2 appears to give a mostly consistent description of $M 1$ properties in rare-earth nuclei, it is also argued that $M 1$ data are really sensitive to single particle degrees of freedom and should not be used to 
determine collective variables [15]. From this point of view, observation of dynamic shape transitions (or lack of it) would help to determine the vector parameters in an independent way, and hence it would provide a significant test for the collective model interpretation of the $M 1$ observables used in the IBM-2. Structurally, the quadrupole parameters used for ${ }^{192} \mathrm{Os}\left(\chi_{\pi}=-0.68, \chi_{\nu}=0.32\right)$ imply competing prolate and oblate shapes for the proton and neutron fluids, respectively. This picture is similar to the pairing-plus-quadrupole model calculations [14], which first predicted the static prolate-oblate shape-phase transition in Os-Pt nuclei.

The errors in the measured quadrupole moments of Os-Pt isotopes [11] are generally too large to reach a definite conclusion whether they actually decrease in absolute value with increasing spin. More precise measurements of the quadrupole moments extending to higher spins are needed to test this prediction. Such measurements are now possible with the new $4 \pi$ high-resolution detector systems "Euroball" and "Gamma-Sphere". It would be very interesting to see whether collective nuclei actually exhibit dynamic shape transitions driven by differences between macroscopic shape variables. Apart from that, they would also provide an important consistency check on the $F$-spin breaking mechanism used in explaining the $M 1$ properties in the IBM-2.

S.K. thanks the members of the nuclear theory group at the University of Tokyo, where this research was carried out, for their hospitality. This work was supported in part by the Australian Research Council and the Department of Industry, Science and Technology, and in part by the Japanese Ministry of Education, Science and Culture. 


\section{References}

[1] J.L. Wood et al., Phys. Rep. 215 (1992) 101.

[2] S. Kuyucak, V-S. Lac and I. Morrison, Phys. Lett. B 263 (1991) 146; S. Kuyucak, Prog. Part. Nucl. Phys. 28 (1992) 391.

[3] J.N. Knudson et al., Phys. Rev. Lett. 66 (1991) 1026.

[4] A.E. Stuchbery et al., Nucl. Phys. A 435 (1985) 635; Nucl. Phys. A 528 (1991) 447; Z. Phys. A 342 (1992) 373.

[5] S. Kuyucak and A.E. Stuchbery, Phys. Lett. B 348 (1995) 315.

[6] T. Otsuka et al., Phys. Lett. B 76 (1978) 139; Nucl. Phys. A 309 (1978) 1.

[7] F. Iachello and A. Arima, The interacting boson model (Cambridge University Press, Cambridge, 1987); T. Otsuka, in: Algebraic approaches to nuclear structure, ed. R.F. Casten (Harwood Academic Publishers, Switzerland, 1993) p. 195.

[8] S. Kuyucak and I. Morrison, Ann. Phys. (NY) 181 (1988) 79; Ann. Phys. (NY) 195 (1989) 126.

[9] T. Otsuka and N. Yoshida, program NPBOS, report JAERI-M85-094.

[10] J.N. Ginocchio and M.W. Kirson, Nucl. Phys. A 350 (1980) 31.

[11] C.Y. Wu et al., Nucl. Phys. A 607 (1996) 178.

[12] S.C. Li and S. Kuyucak, Nucl. Phys. A 604 (1996) 305.

[13] V-S. Lac and S. Kuyucak, Nucl. Phys. A 539 (1992) 418.

[14] K. Kumar, Phys. Scr. 6 (1972) 270.

[15] A. Leviatan, J.N. Ginocchio and M. Kirson, Phys. Rev. Lett. 65 (1990) 2853. 


\section{Figure captions}

Fig. 1. Contour plots of the energy surface (8) in the $\beta_{\pi}-\beta_{\nu}$ plane for a typical deformed nucleus (top) and transitional nucleus (bottom) with $N_{\pi}=8, N_{\nu}=4$ bosons. The parameters are (in $\mathrm{MeV}$ except for $\chi) \varepsilon_{\mathrm{v}}=0, \kappa=0.15, \xi=0.17$, $\chi_{\mathrm{v}}=2$ for both, and $\varepsilon_{\mathrm{s}}=0.2, \chi_{\mathrm{s}}=-0.5$ in the deformed and $\varepsilon_{\mathrm{s}}=0.4, \chi_{\mathrm{s}}=-0.2$ in the transitional case. The prolate (absolute) minima are in the first quadrant and the oblate ones in the third. The contour lines are separated by $1 \mathrm{MeV}$, and the absolute minima are $E_{\min }=-4.83 \mathrm{MeV}$ (top), $E_{\min }=-3.07 \mathrm{MeV}$ (bottom).

Fig. 2. Systematic study of the yrast quadrupole moments in a transitional nucleus against the $F$-spin vector parameters; $N_{\mathrm{v}}(\mathrm{a}), \chi_{\mathrm{v}}(\mathrm{b})$, and $\varepsilon_{\mathrm{v}}(\mathrm{c})$. The scalar parameters are as in Fig. 1 (bottom). The vector parameters are $\chi_{\mathrm{v}}=2, \varepsilon_{\mathrm{v}}=0$ (a), $N_{\mathrm{v}}=4, \varepsilon_{\mathrm{v}}=0(\mathrm{~b})$, and $N_{\mathrm{v}}=4, \chi_{\mathrm{v}}=2(\mathrm{c})$. The lines are drawn to guide the eye.

Fig. 3. Proton-neutron decomposition of the yrast quadrupole moments for the two cases with $\chi_{\mathrm{v}}= \pm 2$ in Fig. $2 \mathrm{~b}$. The other vector parameters are $N_{\mathrm{v}}=4$ and $\epsilon_{\mathrm{v}}=0$. The proton and neutron contributions are indicated by open circles, and the total quadrupole moment by filled circles. The lines are drawn to guide the eye.

Fig. 4. Comparison of the energies, E2 transitions and quadrupole moments of the ground band states in ${ }^{192}$ Os calculated in IBM-2 (solid lines) with the experimental data (circles) [11. The parameters (taken from Ref. [5]) are; $N_{\pi}=3, N_{\nu}=5$, $\varepsilon_{\mathrm{s}}=0.4, \varepsilon_{\mathrm{v}}=0, \kappa=0.15, \chi_{\mathrm{s}}=-0.18, \chi_{\mathrm{v}}=-1, \xi=0.17$ (in MeV except for $N$ and $\chi$ ), and $e_{\pi}=e_{\nu}=0.15 e b$. The results for an axial rotor with $Q_{0}=4.6 \mathrm{eb}$ are also shown for comparison (dashed line). 